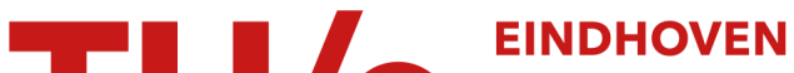 \\ UNIVERSITY OF \\ TECHNOLOGY
}

\section{Resonant enhancement of turbulent energy dissipation}

\section{Citation for published version (APA):}

Cekli, E. H., Tipton, C. R., \& Water, van de, W. (2010). Resonant enhancement of turbulent energy dissipation. Physical Review Letters, 105(4), 1/4-. [044503]. https://doi.org/10.1103/PhysRevLett.105.044503

DOI:

10.1103/PhysRevLett.105.044503

Document status and date:

Published: 01/01/2010

\section{Document Version:}

Publisher's PDF, also known as Version of Record (includes final page, issue and volume numbers)

\section{Please check the document version of this publication:}

- A submitted manuscript is the version of the article upon submission and before peer-review. There can be important differences between the submitted version and the official published version of record. People interested in the research are advised to contact the author for the final version of the publication, or visit the $\mathrm{DOI}$ to the publisher's website.

- The final author version and the galley proof are versions of the publication after peer review.

- The final published version features the final layout of the paper including the volume, issue and page numbers.

Link to publication

\section{General rights}

Copyright and moral rights for the publications made accessible in the public portal are retained by the authors and/or other copyright owners and it is a condition of accessing publications that users recognise and abide by the legal requirements associated with these rights.

- Users may download and print one copy of any publication from the public portal for the purpose of private study or research.

- You may not further distribute the material or use it for any profit-making activity or commercial gain

- You may freely distribute the URL identifying the publication in the public portal.

If the publication is distributed under the terms of Article 25fa of the Dutch Copyright Act, indicated by the "Taverne" license above, please follow below link for the End User Agreement:

www.tue.nl/taverne

Take down policy

If you believe that this document breaches copyright please contact us at:

openaccess@tue.nl

providing details and we will investigate your claim. 


\title{
Resonant Enhancement of Turbulent Energy Dissipation
}

\author{
Hakki Ergun Cekli, Carl Tipton, and Willem van de Water \\ Physics Department, Eindhoven University of Technology, Postbus 513, 5600 MB Eindhoven, The Netherlands
}

(Received 8 April 2010; published 22 July 2010)

\begin{abstract}
We periodically modulate a turbulent wind-tunnel flow with an active grid. We find a resonant enhancement of the mean turbulent dissipation rate at a modulation frequency which equals the largeeddy turnover rate. Thus, we find the best frequency to inject energy into a turbulent flow. The resonant response is characterized by the emergence of vortical structures in the flow and depends on the spatial mode of the stirring grid.
\end{abstract}

DOI: 10.1103/PhysRevLett.105.044503

PACS numbers: 47.27.Gs, 47.27.Jv, 52.25.Gj

Many turbulent flows are subject to periodic modulation. Examples include the flow in an internal combustion engine, the pulsatile blood flow through arteries, and geophysical flows driven by periodic tides. When the modulation is slow, the turbulence will adjust adiabatically; but when the modulation period comes close to an internal time scale of the flow, the turbulence may resonate with the modulation. Such a time scale may be the largeeddy turnover time, or [1] the time needed for the injected energy to cascade down to scales where viscosity reigns. The possibility of a resonance is intriguing, as one may object that turbulence does not have a single dominant time scale, but a continuum of strongly fluctuating times.

Evidence for such a resonant response of turbulence came from simple turbulence models and from direct numerical simulations [1-5]. The turbulence response in these studies, which have inspired the present Letter, was quantified by a shoulder at modulation frequency $f_{m}, f_{m}=$ $f_{r}$ in the response curve, after which the response decays as $f_{m}^{-1}$. The resonance frequency $f_{r}$ was close to the largeeddy turnover rate. In all these studies, the response was quantified through a conditional average at the driving frequency. This is similar to, but not the same as, the spectral energy of a quantity at the driving frequency, which would have demanded prohibitively longintegration times.

Experimental evidence for a response maximum of turbulence was found in the turbulent flow between two counterrotating disks whose rotation rate was varied harmonically [6]. This closed flow allowed a direct measurement of the global energy input rate $\epsilon$, but information about the flow field is quite limited. In these experiment, the behavior of the frequency-selective response was identified as a resonance effect, but the influence on the global energy injection rate was a mere $2.5 \%$.

In this Letter we present the results of an experiment in which turbulence in a wind tunnel is modulated with an active grid which allows us to modulate the flow in space and time. We will, in particular, be interested in the mean rate of energy dissipation $\epsilon$. This contrasts the response of the flow at the driving frequency, to which numerical studies were limited [1-5]. The dependence of $\epsilon$ on the stirring frequency is important for the practical question what the preferred frequency is with which to stir turbulence in order to optimize the energy input.

Active grids, such as the one used in our experiment, were pioneered by Makita [7] and consist of a grid of rods with attached vanes that can be rotated by servo motors. The properties of actively stirred turbulence were further investigated by Mydlarski and Warhaft [8] and Poorte and Biesheuvel [9]. Active grids offer the exciting possibility to tailor turbulence properties by a judicious choice of the space-time stirring protocol [10].

Our grid has mesh size $0.1 \mathrm{~m}$ and consists of 17 axes whose instantaneous angles $\alpha_{i}(t), i=1, \ldots, 17$ are set to $\alpha_{i}(t)=\pi f_{m_{i}} t+\varphi_{i}, f_{m_{i}}= \pm f_{m}$, where $f_{m}$ is the modulation frequency and where the phases $\varphi_{i}, i=1, \ldots, 17$ and the sign of $f_{m_{i}}$ determine the spatial pattern of the timeperiodic grid. The control of the angles is such that all $\alpha_{i}(t)$ are prescribed precisely and remain perfectly synchronous over the used integration times (many hours). In turbulence stirred by static grids, the grid transparency is a key parameter, for example, the classic work by Comte-Bellot and Corrsin concluded that the anisotropy of the velocity fluctuations was smallest for grid transparency $S=0.66$ [11]. Therefore we will characterize the grid state by the time-dependent grid transparency $S(t)$ which follows from the angles $\alpha_{i}(t)$.

The active grid is placed in the $8 \mathrm{~m}$ long experimental section of a recirculating wind tunnel. Turbulent velocity fluctuations are monitored at a distance $D=4.62 \mathrm{~m}$ downstream from the grid using an array of hot-wire anemometers. In the coordinate system where the $x$ axis points in the direction of the mean flow and where the $y$ axis is parallel to the long side of the wind-tunnel cross section (Fig. 1), the turbulent velocity field is represented by the measured $x$ component of the velocity $u\left(y_{i}, t\right)$ at 10 different $y$ positions, $y_{i}, i=1, \ldots, 10$.

Each of the locally manufactured hot wires had a sensitive length of $200 \mu \mathrm{m}$, which is comparable to the smallest length scale of the flow (the measured Kolmogorov scale is $\eta=190 \mu \mathrm{m}$ ). They were operated at constant temperature using computer controlled anemometers that were also developed locally. Each experiment was preceded by a 
calibration procedure in which the voltage to air velocity conversion for each wire was measured using a calibrated nozzle. The resulting 10 calibration tables were updated regularly during the run to allow for a (small) temperature increase of the air in the wind tunnel. The signals of the sensors were sampled exactly simultaneously at $20 \mathrm{kHz}$, after being low-pass filtered at $10 \mathrm{kHz}$. At each modulation frequency, time series were acquired that typically contained $2 \times 10^{6}$ integral scales. Simultaneous with the wind velocity data, the angles of the grid were registered so that the correlation between the grid and the turbulence could be computed. The typical mean velocity in our experiments is $U=9 \mathrm{~ms}^{-1}$, the fluctuating velocity $u=1 \mathrm{~ms}^{-1}$, while a typical Reynolds number $\operatorname{Re}_{\lambda}=500$ resulted in fairly sized inertial range.

Our active grid can be used to impose a large variety of patterns, but they are subject to the constraint that a single axis drives an entire column of vanes. In Fig. 1 we show the snapshots of a time-periodic grid spatial mode that produced the results described in this Letter. The phasesensitive average of the turbulent velocity shown in Fig. 1(c) demonstrates that at very low modulation frequencies, the wind follows approximately adiabatically, while at large $f_{m}$ the turbulence can no longer follow the modulation.

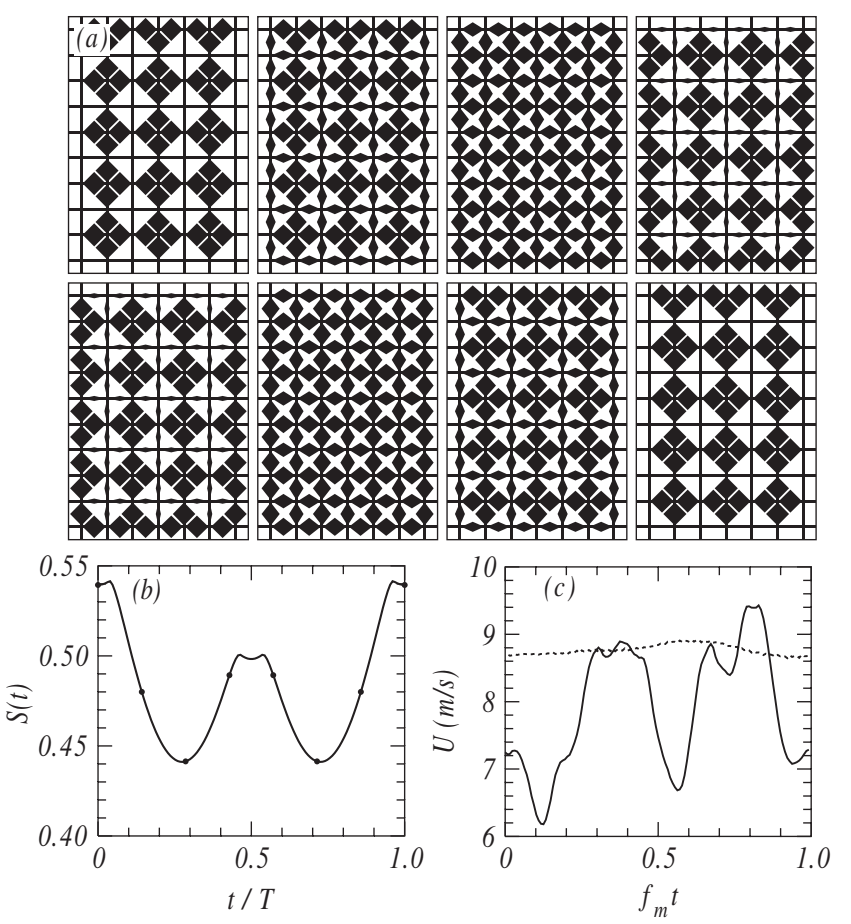

FIG. 1. (a) Snapshots of one period of a time-periodic grid mode. This spatial mode is realized by a particular choice of the initial grid phases $\varphi$. The grid fills the $0.7 \times 1.0 \mathrm{~m}^{2}$ cross section of the wind tunnel. (b) One period of the transparency $S(t)$ of the grid, the dots correspond to the snapshots in (a). (c) Full line: phase-averaged response at modulation frequency $f_{m}=1 \mathrm{~Hz}$, dashed line at $f_{m}=10 \mathrm{~Hz}$. At the largest frequency the turbulence can no longer follow the modulation.
We quantify the response of the turbulent flow in several ways. Most straightforwardly, we measure the timeaveraged energy dissipation rate $\epsilon$ as a function of the modulation frequency $f_{m}$. As the small-scale dissipation rate equals the energy input per unit of mass and time, measuring its modulation frequency dependence can answer the question whether there is an optimum frequency to stir the flow. Next, we will study time-dependent quantities at the modulation frequency. For studying the correlation between the fluctuations of $\epsilon$ and the state of the grid, we are interested in the time dependence of $\epsilon(t)$ on the time scale of the modulation, which is 2 orders of magnitude slower than the turbulence time scales that contribute most to $\epsilon$. Assuming isotropy, the (pseudo-) energy dissipation rate $\epsilon(t)$ was inferred from a single derivative, $\epsilon(t)=15 \nu\left\langle(\partial u / \partial x)^{2}\right\rangle_{y}$, with $\nu$ the kinematic viscosity of air and where a spatial average was done over the extent in $y$ of the probe array. Taylor's frozen turbulence hypothesis was used to infer the spatial separation $x$ from time delays through $x=U t$, with $U$ the mean velocity. Alternatively, the mean energy dissipation rate $\langle\epsilon\rangle_{t}$ follows from the measured energy spectrum, $E(k)=c_{K} \epsilon^{2 / 3} \kappa^{-5 / 3}$, with the Kolmogorov constant $c_{K}=0.53$ [12].

More detailed information about the response of turbulence can be obtained from the normalized correlation of the grid state $S(t)$ and the turbulent velocity $u(t), C(\tau)=$ $\langle S(t+\tau) u(t)\rangle$, normalized such that $C(\tau=0)=1$, and $C(\tau \rightarrow \infty)=0$. In numerical simulations, the modulation and turbulent response spatially coincide. However, this is not so in the experiments where a mean wind advects the modulation. In our case this leads to a trivial convective time delay $\tau_{c}=D / U$. From the zero crossings $\tau_{0}$ of the correlation function, the relative phase between the periodic stirring and the turbulence can then be determined as $\phi=2 \pi f_{m}\left(\tau_{0}-\tau_{c}\right)$.

A typical trace of $\epsilon(t)$ with the grid driven at $f=4 \mathrm{~Hz}$ is shown in Fig. 2, together with the periodic grid transpar-

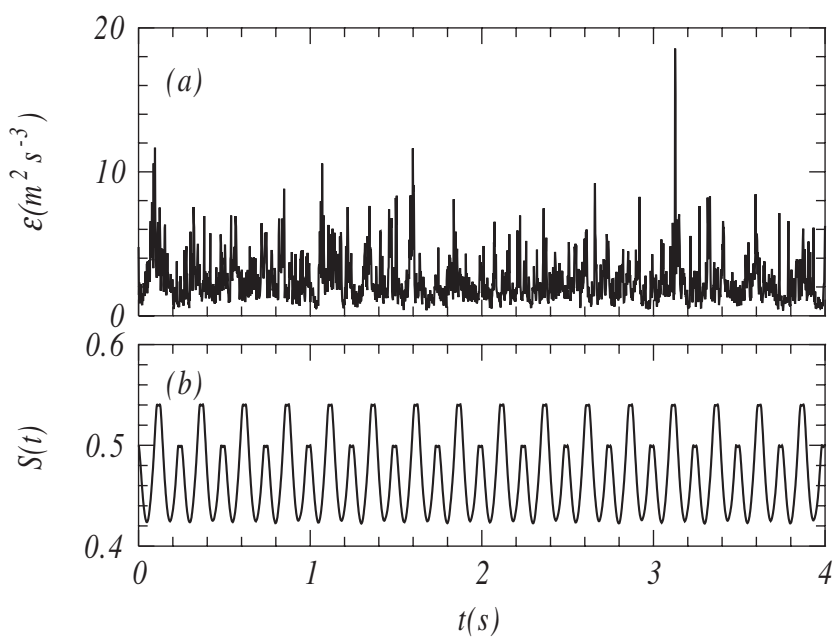

FIG. 2. (a) Trace of $\epsilon(t)$ with the grid driven at $f_{m}=4 \mathrm{~Hz}$. The dissipation rate $\epsilon(t)$ is low-pass filtered at $5 \times 10^{2} \mathrm{~Hz}$ $(83 \eta)$. (b) Grid transparency $S(t)$. 
ency. Although the periodic modulation can hardly be traced in $\epsilon(t), S$ and $\epsilon$ are strongly correlated with $C(\tau)$ having amplitude $\approx 0.3$.

The frequency dependence of the mean dissipation rate $\epsilon$ is shown in Fig. 3(a). It shows a strongly resonant response of the averaged dissipation rate which corresponds to a $50 \%$ enhancement of the injected energy at the reduced frequency $f_{m} T_{L}=1.5$, irrespective of the turbulent intensity. In these experiments the large-eddy turnover time $T_{L}=L / u$, where $u$ is the turbulent velocity, varies by a factor 5 . The resonant response is accompanied by a phase shift $\phi \approx \pi / 2$ between the turbulent velocity and the grid state $S$. The integral scale $L$ was determined from the spectrum $E(f)$ as $L=U E_{a}(f=0) / 2 u^{2}$. We find that $L=0.21 \pm 0.02 \mathrm{~m}$, independent of the modulation frequency. The integral scale is approximately twice the mesh size of the active grid.

The observation of this resonance with its companion phase shift was anticipated by numerical simulations [4], but the resonant enhancement that is measured here is much stronger; it is also observed in an averaged quantity, and not just in the response at the modulation frequency.

Let us now consider a measurement of the response in a manner that resembles that used in numerical simulations [1-5] and other experiments [6]. In Fig. 4 we show measured energy spectra $E\left(f, y_{i}\right)$ at the location $y_{i}$ of the velocity probes. All spectra display a well-developed inertial range behavior that is the same for all $y_{i}$. They are marred by spikes at the modulation frequency and its harmonics whose height decreases for increasing driving frequency $f_{m}$, but in a way that depends on $y$.

The frequency-selective response $R\left(f_{m}, y_{i}\right)$ is defined as the ratio of the energy in these spikes and the total turbulent energy $u^{2}$ [see Fig. 4(c)]. The frequency dependence of the spatial average $R_{a}$ of $R\left(f_{m}, y\right), R_{a}\left(f_{m}\right)=\left\langle R\left(f_{m}, y\right)\right\rangle_{y}$, and
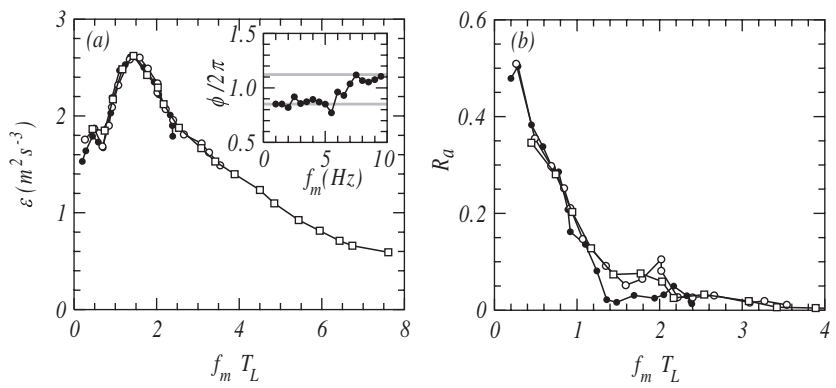

FIG. 3. (a) Mean dissipation rate $\epsilon$, (b) Frequency-selective response $R_{a}$ as a function of the dimensionless modulation frequency $f_{m} T_{L}$, with $T_{L}$ the large-eddy turnover time. The dots, circles and squares correspond to mean velocities $U=9$, 6 , and $4 \mathrm{~ms}^{-1}$, respectively. The $\epsilon$ corresponding to $U=6$, and $4 \mathrm{~ms}^{-1}$ has been scaled such that the maximum coincides with that at $U=9 \mathrm{~ms}^{-1}$. At large dimensionless frequencies, the dissipation rate approaches that of the open grid. Inset in (a): relative phase $\phi$ between the grid state $S(t)$ and the turbulent velocity $u(t)$. The mean velocity is $U=9 \mathrm{~ms}^{-1}$. At resonance, $f_{m} \approx 6 \mathrm{~Hz}\left(f_{m} T_{L} \approx 1.5\right)$, the phase jumps by $\approx \pi / 2$. the spatial dependence of $R\left(f_{m}, y\right)$ are shown in Figs. 3(b) and 4(b), respectively. The response $R_{a}\left(f_{m}\right)$, both at the driving frequency and its first harmonic, rapidly decreases for frequencies beyond the large-eddy turnover frequency $T_{L}^{-1}$. Both numerical simulations [1-5] and other experiments [6] find an amplitude response that decays with frequency as $1 / f_{m}$, for our definition this implies $R_{a}\left(f_{m}\right) \sim$ $f_{m}^{-2}$; our results roughly follow this trend.

It must be realized that its time- and space dependent transparency does not uniquely determine the motion of an active grid. In fact, the sequence of patterns shown in Fig. 1(a) can be realized with different rotation senses of the axes. With the restraint that neighboring axes run in opposite directions, there are 4 different ways to make Fig. 1(a). Of these 4 possible modes, there is only one that produces the resonances of Fig. 3. The other modes have resonances that are much less pronounced, and occur at lower frequencies.

The spatial dependence of the response $R\left(f_{m}, y\right)$ in Fig. 4 and the influence of the spatial structure of the stirring grid suggest that the resonant enhancement of the turbulence energy input proceeds through structures of the velocity field. A map of the velocity field measured in the $x-y$ plane centered in the wind tunnel was made using particle-image velocimetry (PIV). The $(u, v)$ components of the velocity field were obtained by measuring the displacement of small particles between two subsequent images of the flow in a thin laser sheet. Both the flashes of the laser and the acquisition of the images were synchronized with the periodic motion of the grid.

The phase-averaged velocity fields are shown in Fig. 5 at frequencies $f_{m}=1,6$ and $10 \mathrm{~Hz}$. At the used mean velocity $U=9 \mathrm{~ms}^{-1}$, the resonance would be at $f_{m}=6 \mathrm{~Hz}$ $\left(f_{m} T_{L}=1.5\right)$. Indeed, at this frequency a marked vortical structure is observed in Fig. 5(b), which spans approxi-

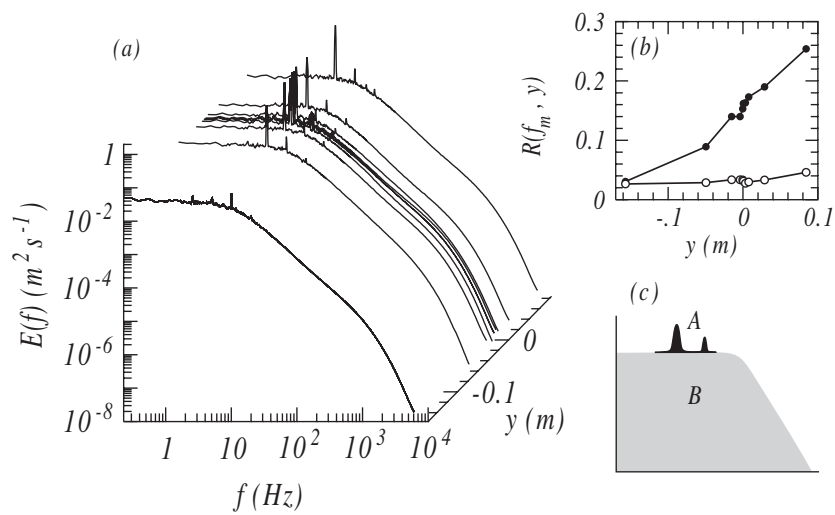

FIG. 4. (a) Energy spectra $E\left(f, y_{i}\right)$ at the probe positions $y_{i}$, $i=1, \ldots, 10$. The grid modulation frequency is $f_{m}=5 \mathrm{~Hz}, y=$ 0 refers to the center of the wind-tunnel cross section. (b) Spatial dependence of the response $R\left(f_{m}, y\right)$ at modulation frequencies that straddle the resonance, open circles: $f_{m}=6.5 \mathrm{~Hz}$, closed dots: $f_{m}=4.5 \mathrm{~Hz}$. (c) The response is defined as the ratio of the energy in the peaks $A$ at $f_{m}$, and $2 f_{m}$, divided by the total energy $A+B$. 

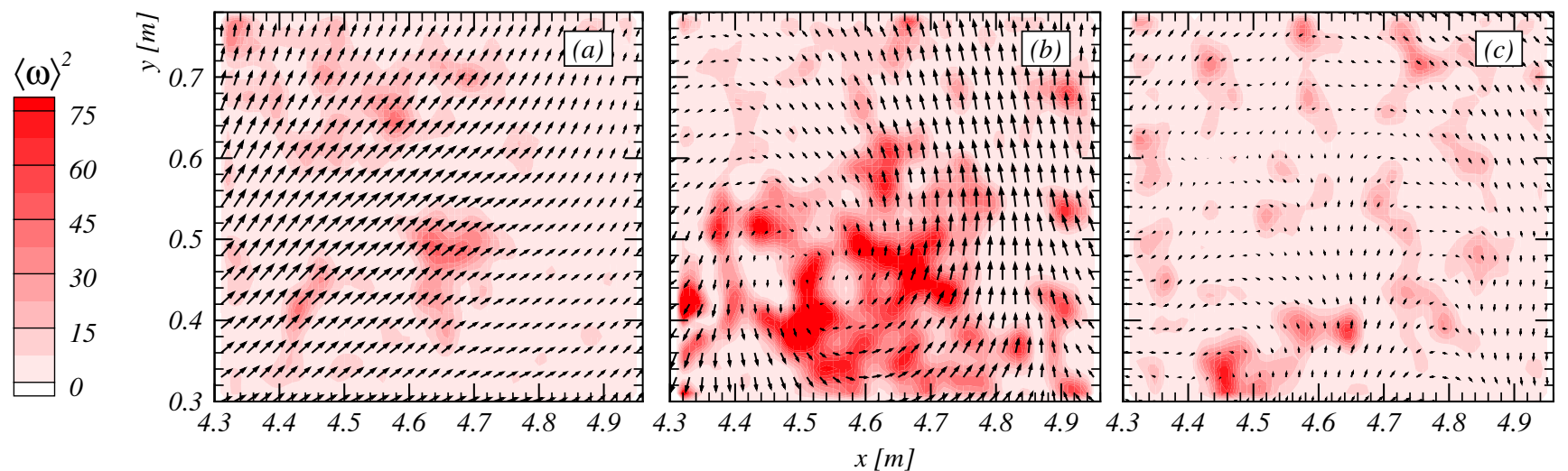

FIG. 5 (color online). Phase-averaged velocity fields at 3 modulation frequencies, $f_{m}=1,6$, and $10 \mathrm{~Hz}$ for frames (a), (b) and (c), respectively. The velocity components are indicated by arrows, the squared mean vorticity field $\left\langle\omega_{z}\right\rangle^{2}=\langle\partial v / \partial x-\partial u / \partial y\rangle^{2}$ is indicated by the color scale. The mean velocity $U=9 \mathrm{~ms}^{-1}$ was subtracted. After a correction for the finite resolution of the PIV measurement [14], and assuming isotropy, the dissipation rate can be estimated by $\epsilon=15 / 4 \nu\left\langle\omega_{z}^{2}\right\rangle$.

mately the integral length scale. Although the space-time resolution of particle-image velocimetry cannot rival that of hot-wire probes, a crude estimate of $\epsilon$ based on the entire span of the velocity field shown in Fig. 5, agrees with the results shown in Fig. 3(a).

In our experiment, the strong resonant enhancement of $\epsilon$ leaves the small-scale turbulent intermittency unaffected. The probability density function (PDF) of velocity increments $u(y+d y)-u(y)$, measured over a spatial separation $d y$, only depends on the phase of the oscillation at large separations $d y$ and small modulation frequencies $f_{m}$, while the cycle-averaged PDF at $d y / \eta=4$, does not depend on $f_{m}$. This is in contrast with [13], where a slowly precessing vortex between two corotating disks was found to influence the shape of the PDF at small scales, and thus the turbulent cascade.

In conclusion, we have found a resonant enhancement of turbulent dissipation in a time-periodically modulated wind-tunnel flow. The details of the turbulence response depend on the spatial structure of the stirrer. Modulation of turbulence acts at low frequencies and large scales; the small-scale structure responds only indirectly through the energy dissipation rate. The filtering action of turbulence, which is not well understood, prevents large modulation frequencies from invading the inertial range. This may explain the success of large-eddy computations for predicting these phenomena numerically [4]. Finding the optimal way to stir turbulence is of enormous practical importance. The stirring frequency should match the large-eddy turnover rate, but the question remains how to design the optimal spatial stirring pattern.

This work is part of the research programme of the "Stichting voor Fundamenteel Onderzoek der Materie
(FOM)", which is financially supported by the "Nederlandse Organisatie voor Wetenschappelijk Onderzoek (NWO)." EU support was through contract HPRN-CT-2002-00300 and COST Action MP0806. We thank Freek van Uittert, Gerald Oerlemans, and Ad Holten for technical assistance.

[1] A. von der Heydt, S. Grossmann, and D. Lohse, Phys. Rev. E 67, 046308 (2003).

[2] A. von der Heydt, S. Grossmann, and D. Lohse, Phys. Rev. E 68, 066302 (2003).

[3] A. K. Kuczaj, B. J. Geurts, and D. Lohse, Europhys. Lett. 73, 851 (2006).

[4] W. J. T. Bos, T. T. Clark, and R. Rubinstein, Phys. Fluids 19, 055107 (2007).

[5] A. K. Kuczaj, B. J. Geurts, D. Lohse, and W. van de Water, Comput. Fluids 37, 816 (2008).

[6] O. Cadot, J.H. Titon, and D. Bonn, J. Fluid Mech. 485, 161 (2003).

[7] H. Makita, Fluid Dyn. Res. 8, 53 (1991).

[8] L. Mydlarski and Z. Warhaft, J. Fluid Mech. 320, 331 (1996).

[9] R. E. G. Poorte and A. Biesheuvel, J. Fluid Mech. 461, 127 (2002).

[10] H.E. Cekli and W. van de Water, Exp. Fluids 49, 409 (2010).

[11] G. Comte-Bellot and S. Corrsin, J. Fluid Mech. 25, 657 (1966).

[12] B. R. Pearson, P.-A. Krogstad, and W. van de Water, Phys. Fluids 14, 1288 (2002).

[13] R. Labbé, J.-F. Pinton, and S. Fauve, Phys. Fluids 8, 914 (1996).

[14] J. Sheng, H. Meng, and R. Fox, Chem. Eng. Sci. 55, 4423 (2000). 